\title{
Rogue wave fission
}

\author{
Amdad Chowdury $\odot$ and Wonkeun Chang $\odot$ \\ School of Electrical and Electronic Engineering, Nanyang Technological University, 639798, Singapore
}

(Received 29 January 2021; accepted 25 August 2021; published 8 September 2021)

\begin{abstract}
We investigate the effect of the third-order dispersion on higher-order rogue wave solutions. We find that under the presence of weak perturbation, the higher-order rogue wave breaks apart into its fundamental rogue wave constituents, similar to how a higher-order soliton undergoes a fission. Each breakaway rogue wave solution is separated in space and time and is accompanied by emission of a dispersive wave.
\end{abstract}

DOI: 10.1103/PhysRevResearch.3.L032060

\section{INTRODUCTION}

The discovery of the soliton solutions for certain types of nonlinear evolution equations marks an important milestone in mathematical physics. Among many types of evolution equations, the nonlinear Schrödinger equation (NLSE) takes center stage in science and engineering, not only for its applications but also for its ubiquitous nature. In physics, NLSE and its solutions have been applied in optics, plasma physics, hydrodynamics, condensed matter physics, and Bose-Einstein condensates [1-4], while in mathematics they are studied in integrability, Riemann-Hilbert problems, as well as in the theory of solitons and rogue waves [5,6].

The successful implementation of the inverse scattering transformation on NLSE by Zakharov and Shabat led to the soliton solutions in their exact mathematical forms [7]. A soliton, in its elementary state, is a stable wave that propagates without distortion. Their nonlinear superposition appears as a higher-order soliton, which evolves periodically through mutual interactions. However, when the symmetry is broken due to system perturbation, the soliton bound state collapses, disintegrating into its fundamental soliton parts. This process is called soliton fission, which has been studied extensively in the context of supercontinuum generation in optics and hydrodynamics [8-10].

Other than solitons, a few different families of NLSE solutions have later been discovered with new intriguing properties. The most notable examples in this regard are the breather and rogue wave solutions [11]. They have attracted considerable attention as they can explain interesting albeit complex nonlinear phenomena-modulational instability (MI). Since its first discovery by Benjamin [12], MI became one of the central topics in nonlinear science as a mechanism that drives pattern formation in various systems [13]. MI refers to an exponential amplification of small periodic modulation on a continuous-wave background. In the Fourier domain, this translates to generating a cascade of spectral

Published by the American Physical Society under the terms of the Creative Commons Attribution 4.0 International license. Further distribution of this work must maintain attribution to the author(s) and the published article's title, journal citation, and DOI. sidebands. These sidebands and the original continuous-wave exchange energy, eventually forming a series of pulses which gives rise to the Akhmediev breather solution [14]. This breather solution appears in the form of well-known rogue wave solution as its limiting case when the period in the transverse dimension becomes infinite. In fact, both the rogue wave and the soliton solutions are the limiting cases of three general NLSE solutions, i.e., cnoidal waves, Akhmediev breather, and Kuznetsov-Ma breather [15]. The rogue wave solution describes the sudden appearance of an extreme-amplitude freak wave in the ocean or a burst of light with exceptional intensity in optical fiber [16-18].

Much like solitons, a nonlinear combination of several fundamental rogue wave solutions can create a higher-order rogue wave [19]. The impact of various perturbations on the fundamental rogue wave solution has been studied extensively. For instance, the solution stability for very small values of the third-order dispersion, self-steepening, and Raman self-frequency shift on the fundamental rogue wave has been theoretically investigated [20]. Also, the presence of a fundamental rogue wave in the turbulent wave field has been numerically confirmed in NLSE perturbed with the thirdorder dispersion [21]. On the other hand, there are no studies on the general effect of perturbations on higher-order rogue wave solutions until now. In this work, we take the first step, and study higher-order rogue wave solutions in the presence of the third-order dispersion, which we introduce as a perturbation to NLSE to observe the solution behavior. Physically, the effect of third-order dispersion becomes crucial when the spectrum of the localized wave broadens such that the group-velocity dispersion can no longer be considered fixed across its spectrum [22,23]. We demonstrate that under weak symmetry-breaking perturbation, a higher-order rogue wave solution breaks apart and reduces to its constituent fundamental rogue waves. This fascinating observation bears a striking similarity to soliton fission phenomena.

\section{FUNDAMENTAL ROGUE WAVE SOLUTION}

NLSE in its dimensionless form is given by:

$$
i \psi_{z}+\frac{1}{2} \psi_{t t}+\psi|\psi|^{2}=0
$$



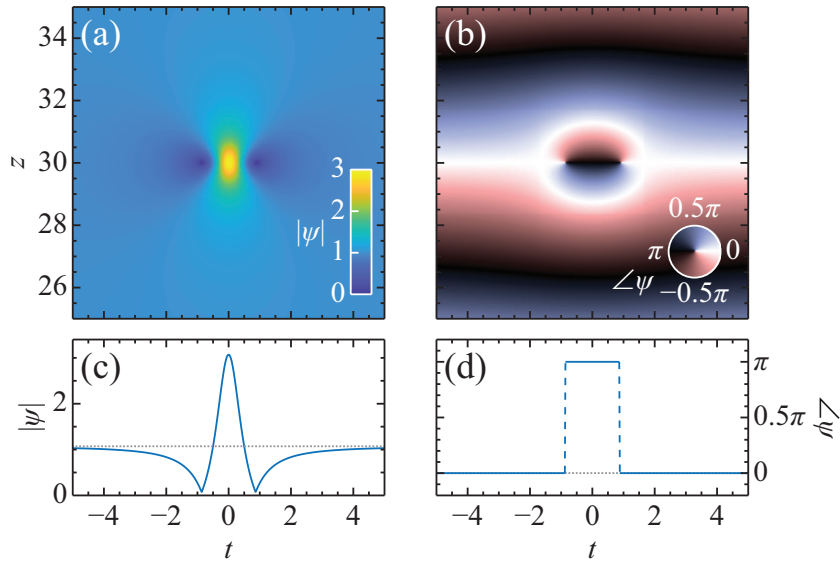

FIG. 1. (a) Amplitude and (b) phase profiles of the fundamental rogue wave solution. Panels (c) and (d) are its profiles at the maximum compression point, i.e., $z=30$. Black-dotted lines indicate the amplitude and phase of the initial condition used at $z=0$ in the numerical simulation.

where $\psi(z, t)$ is the complex envelope of the waves, and $z$ and $t$ are the variables in the propagation and transverse directions, respectively.

Figures 1(a) and 1(b) present the amplitude and phase profiles of the fundamental rogue wave solution. To numerically excite this, we adopt the analytical solution well before its fully developed stage as the initial condition. The solution in its exact form is obtained by solving Eq. (1) through the Darboux transformation technique using a continuous wave, $\psi=\exp (i z)$, as the seed [24]. The initial condition has an infinitesimally small amplitude and almost zero phase shift at $t=0$ as plotted in black-dotted lines in Figs. 1(c) and 1(d). We apply the split-step Fourier method for the numerical simulations. We use a wide, $t_{0}=2000$, and flat-top, $m=$ 20 , super-Gaussian pulse of the form $\exp \left[-0.5\left(t / t_{0}\right)^{2 m}\right]$ to represent a continuous-wave background. The range of the $t$ window is $2000 \pi$ with $2^{20}$ grid points. This ensures the amplitude is almost flat in the central part of the $t$ window, while it is effectively zero at the periodic boundary. MI drives the initial condition into the fundamental rogue wave solution, which at its peak height, at $z=30$, the maximum amplitude reaches 3 and the phase shift across the peak becomes $\pi$. Its amplitude and phase profiles at the maximum compression point are shown in Figs. 1(c) and 1(d).

\section{SECOND-ORDER ROGUE WAVE SOLUTION AND ITS FISSION}

The evolutions of the amplitude and phase profiles of the second-order rogue wave solution around its maximum compression are depicted in Figs. 2(a) and 2(b). This is obtained numerically using the same approach as the fundamental rogue wave in Fig. 1, i.e., by applying the analytical second-order rogue wave solution well before it fully emerges. Figures 2(c) and 2(d) are its amplitude and phase at $z=30$ where the maximum amplitude of 5 is achieved. We note that a second-order rogue wave solution is a result of the nonlinear superposition of three fundamental rogue waves.
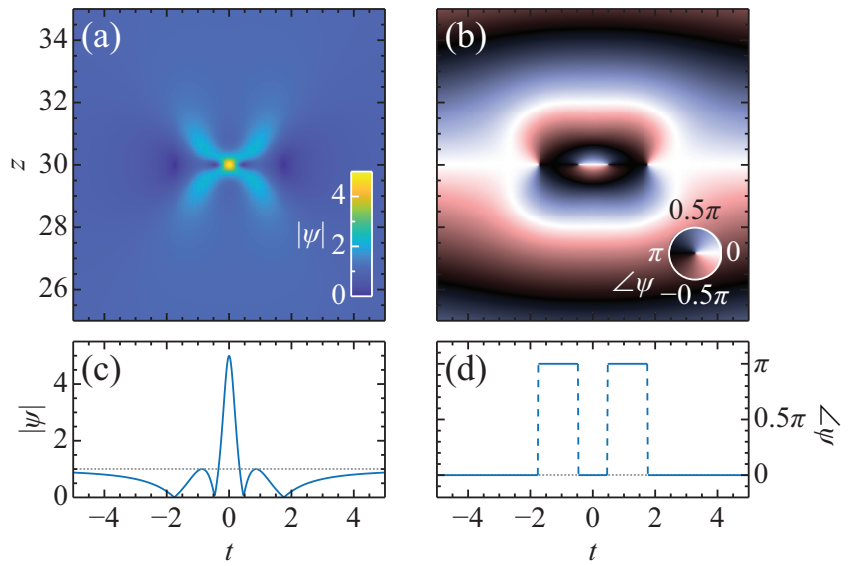

FIG. 2. (a) Amplitude and (b) phase profiles of the second-order rogue wave solution. Panels (c) and (d) are its profiles at the maximum compression point, i.e., $z=30$. Black-dotted lines indicate the amplitude and phase of the initial condition used at $z=0$ in the numerical simulation.

More generally, a higher-order rogue wave solution of order $N$ consists of $N(N+1) / 2$ fundamental rogue waves [25,26].

We introduce the third-order dispersion term as a form of symmetry-breaking perturbation to NLSE. The modified equation is now given by:

$$
i \psi_{z}+\frac{1}{2} \psi_{t t}-i \frac{\beta_{3}}{6} \psi_{t t t}+\psi|\psi|^{2}=0,
$$

where $\beta_{3}$ is the third-order dispersion parameter. The effect of the perturbation on the second-order rogue wave solution is examined numerically by solving Eq. (2) for a given $\beta_{3}$ with the same initial condition as in Fig. 2. Figure 3(a) is the amplitude evolution of the second-order rogue wave solution when $\beta_{3}=0.18$. Note that when $\beta_{3}$ is negative, the solution is simply inverted in $t$. The presence of $\beta_{3}$ prevents the emergence of the second-order rogue wave solution to its full height. Instead, it appears as three fundamental rogue waves separated in $z$ and $t$. This is clearly observable within the red-dashed box in Fig. 3(a), labeled RW1, RW2, and RW3. The amplitude and phase evolutions of the red-highlighted
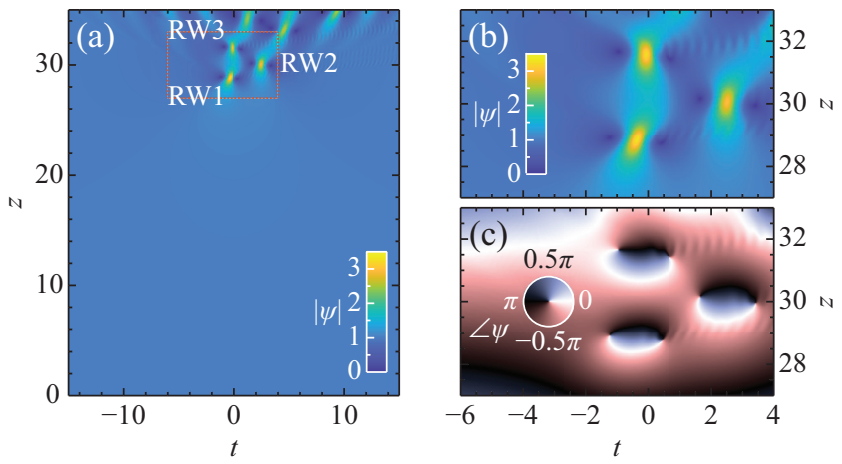

FIG. 3. (a) Breaking of the second-order rogue wave solution under the influence of $\beta_{3}=0.18$. Enlarged evolutions of (b) amplitude and (c) phase profiles around the appearance of the three localized peaks labeled RW1, RW2, and RW3. 

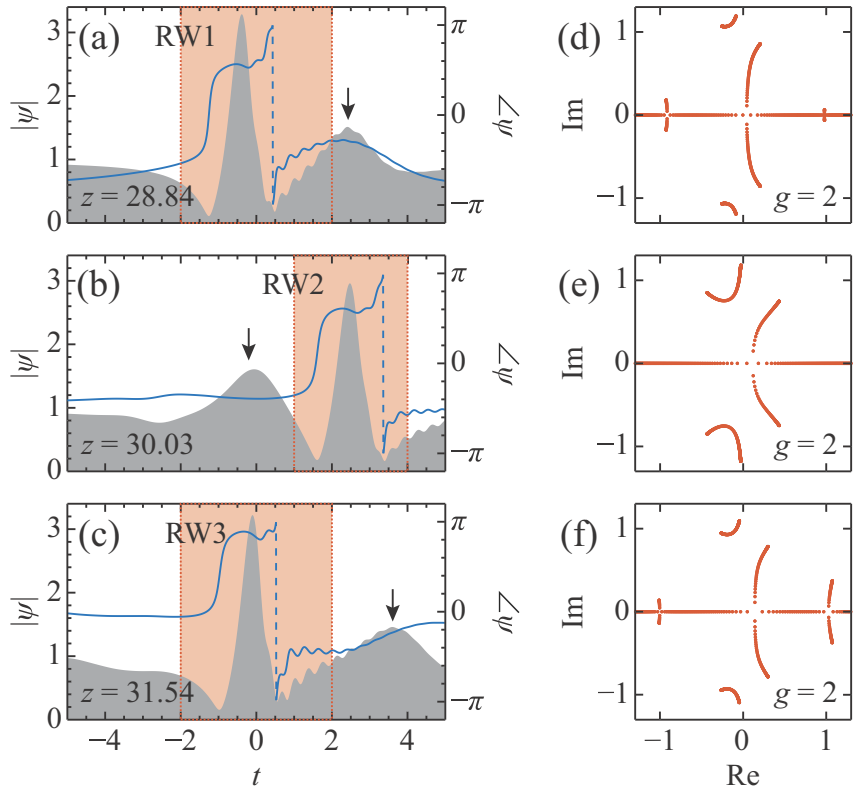

FIG. 4. Amplitude (gray shades) and phase (blue lines) profiles of (a) RW1, (b) RW2, and (c) RW3 at their respective maximum heights. Panels (d), (e), and (f) are the corresponding IST spectra of the solutions in the highlighted sections isolated and periodized.

section are presented in Figs. 3(b) and 3(c). Each of the localized structures has a maximum amplitude of around 3 and undergoes a phase shift of $\pi$ across its peak, which are the primary signatures of the fundamental rogue wave solution.

The amplitude and phase profiles of the disintegrated parts, i.e., RW1, RW2, and RW3, at their respective maximum heights are shown in Figs. 4(a)-4(c). While they resemble those of the fundamental rogue wave solution to a large degree, such as the $\pi$ shifted flat phase at the center of each amplitude peak, there are also noticeable differences that are expected due to the presence of the perturbation. In particular, the appearance of the individual rogue wave component is accompanied by a linear wave radiation. It is seen as a fastoscillating modulation that sits on the right-hand side of the amplitude peak in the case of positive $\beta_{3}$. As we shall see, this is the dispersive wave generated from the fundamental rogue wave under the influence of the perturbation. The bulges, marked with arrows, that are observed next to the amplitude peaks in Figs. 4(a)-4(c) are the appearing or disappearing instances of the subsequent or earlier fundamental rogue waves.

For each numerical snapshot in Figs. 4(a)-4(c), we perform an inverse scattering transform (IST) spectral analysis. The highlighted formation is isolated and periodized to get a discrete IST spectrum for classifying the structure as shown in Figs. 4(d)-4(f). The technique to obtain the spectrum and its classification is detailed in Randoux et al. [27]. All three snapshots exhibit three spectral bands around the imaginary axis in each, featuring the genus, $g=2$, which confirms that they belong to the class of rogue wave solutions. The symmetry of the spectrum that is expected in the unperturbed NLSE is clearly broken due to the presence of $\beta_{3}$.

The details of the evolution dynamics are subject to the magnitude of $\beta_{3}$. When it is small, i.e., $\left|\beta_{3}\right| \lesssim 0.04$, the in-
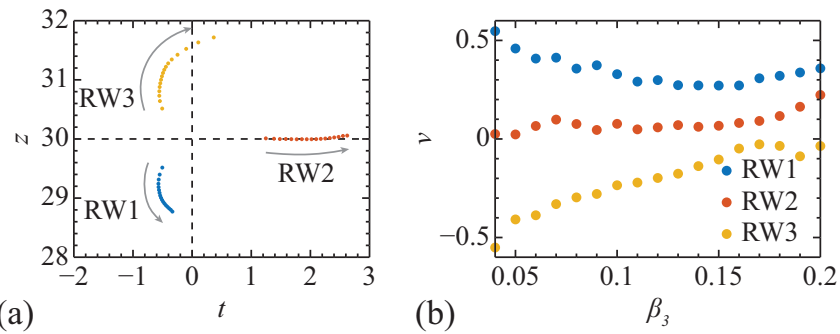

FIG. 5. (a) Positions of the peaks of the rogue wave components as $\beta_{3}$ is varied. The arrows are in the directions of increasing $\beta_{3}$. (b) Instantaneous velocity, $v=d t / d z$, for each rogue wave against $\beta_{3}$.

dividual rogue wave components are not fully separated and remain indistinguishable. For $0.04 \lesssim\left|\beta_{3}\right| \lesssim 0.2$, three fundamental parts of the second-order rogue wave solution are visually observable. $\beta_{3}$ imposes a velocity on the solution in the direction of positive $t$, which is evident from the general skew of the evolving structures toward the right-hand side. However, at the points of their appearance, the disintegrated rogue waves are not aligned to this $\beta_{3}$-induced tilt. Shown in Fig. 5(a) are the positions of the three constituent rogue waves as $\beta_{3}$ is changed, where the arrows denote the directions of increasing $\beta_{3}$. The corresponding instantaneous velocities, $v=d t / d z$, at the moments of their emergence are presented in Fig. 5(b). Each component takes its independent path as $\beta_{3}$ is increased, widening the peak-to-peak distances among them. In the initial development stage of the second-order rogue wave solution, the effect of $\beta_{3}$ is caught up by each constituent fundamental rogue wave at different points in $z$ and $t$. This induces translations among the components with varying degrees of velocities. Beyond $\left|\beta_{3}\right| \gtrsim 0.2$, the effect of the third-order dispersion is too strong for the disintegrated rogue waves to show up. Rather, the higher-order rogue wave solution directly transforms into a group of comparatively low amplitude solitons. To this end, we refer to past studies which demonstrate that a higher-order soliton and fundamental rogue wave solutions share near identical temporal and spectral profiles at their maximum compression points [28,29]

In the presence of $\beta_{3}$, each disintegrated rogue wave emits a linear wave. This is the dispersive wave associated with the corresponding fundamental rogue wave which is addressed comprehensively in a recent work for both weakly and strongly perturbed systems [30]. It reports that under the weak effect of $\beta_{3}$, a fundamental rogue wave emits a phase-matched linear wave only at a single frequency, whereas when $\beta_{3}$ becomes stronger, multiple radiations at different frequencies are observed. Figure 6(a) shows the spectral evolution around the emergence of the three rogue waves when $\beta_{3}=0.18$. It is for the same system featured in Figs. 3(b) and 3(c). The onset of the frequency up-shifted dispersive waves at the moments of the fundamental rogue wave appearance, marked with three horizontal-dashed lines, is clearly visible. The angular frequencies of the emission peaks, $\omega_{1}, \omega_{2}$, and $\omega_{3}$, are determined from the numerical results for a range of $\beta_{3}$ values. This is summarized in Fig. 6(b). 

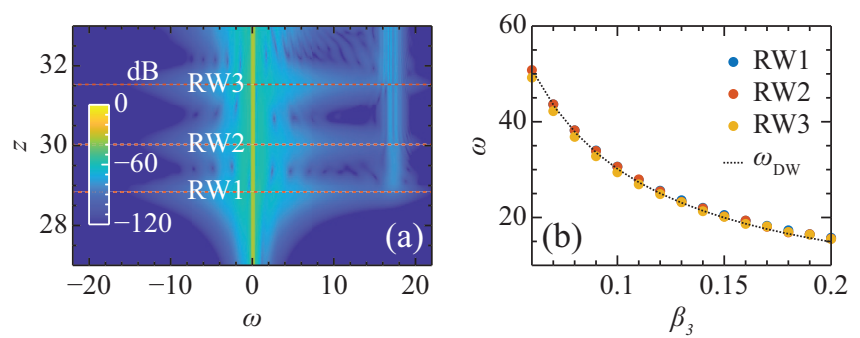

FIG. 6. (a) Spectral evolution around the emergence of the three rogue waves when $\beta_{3}=0.18$. It is for the same system featured in Figs. 3(b) and 3(c). The horizontal-dashed lines mark the positions of the three fundamental rogue waves at their maximum heights. (b) Frequencies of the dispersive waves measured numerically at the peak of each rogue wave appearance versus $\beta_{3}$. The fundamental rogue wave phase-matching frequency, $\omega_{\mathrm{DW}}$, approximated using Eq. (3) is shown together for reference.

The phase-matching frequency, $\omega_{\mathrm{DW}}$, can be approximated to the first order to be:

$$
\omega_{\mathrm{DW}}=3 / \beta_{3},
$$

which is plotted in Fig. 6(b). The numerically observed radiations match closely the approximation obtained in Eq. (3) and are commensurate with the earlier report made on a single fundamental rogue wave solution [30]. This further validates that RW1, RW2, and RW3 are indeed the fundamental rogue waves.

\section{THIRD- AND HIGHER-ORDER ROGUE WAVE FISSION}

The same concept can be extended to $N$-th-order rogue wave solutions. As an example, we apply $\beta_{3}$ on a third-order rogue wave, which from the exact analytical solution is known to consist of six fundamental rogue wave solutions. We take the same numerical approach as in the previous case. Shown in Figs. 7(a) and 7(b) are the amplitude and phase evolutions of the third-order rogue wave solution with $\beta_{3}=0.15$ in the vicinity of its appearance. The perturbation breaks apart the structure into a cluster of six fundamental rogue waves. The same applies to all $N$-th-order rogue wave solutions.

From the splitting of higher-order rogue wave solutions into their elementary components, we can draw a direct analogy with higher-order soliton fission. In soliton fission, an $N$-th-order soliton, i.e., $\psi=N \operatorname{sech}(t)$, breaks into its constituent fundamental $N$ solitons, each with different ampli-
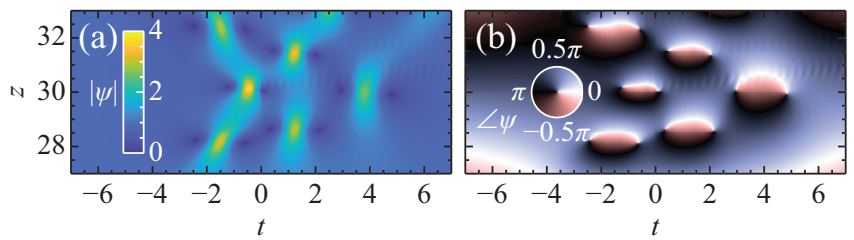

FIG. 7. (a) Amplitude and (b) phase evolutions showing the effect of $\beta_{3}=0.15$ on a third-order rogue wave solution in the vicinity of its appearance. Each disintegrated component clearly exhibits the characteristics of a fundamental rogue wave and emits a dispersive wave. tude, phase, and duration. This is much like the disintegration of a higher-order rogue wave solution into its basic parts as we have shown in this work. Furthermore, our preliminary study reveals that the fission can also be observed in the presence of other symmetry-breaking perturbations such as the selfsteepening effect. This is proof that the fission of nonlinear coherent structures is a commonly observed phenomenon.

We also note differences in their evolution dynamics. MI is the principal mechanism for the emergence of rogue wave solutions, while for solitons this is not the case. Instead, the combined effect of anomalous dispersion and self-phase modulation forms the soliton. It is a stable wave that propagates on a zero background, and hence when $\beta_{3}=0$, both the fundamental and the higher-order solitons remain intact. In fact, the former prevails even when $\beta_{3} \neq 0$, whereas the higherorder solitons become unstable leading to the soliton fission. In contrast, both the higher-order and the fundamental rogue wave solutions are highly unstable structures of NLSE with or without $\beta_{3}$. Unlike the soliton that propagates steadily, a rogue wave solution emerges momentarily on top of a continuous wave. $N(N+1) / 2$ fundamental rogue waves seeded from the initial condition are subject to an exponential growth due to MI. However, while they are developing, $\beta_{3}$ breaks the symmetry of the process and stimulates asymmetric translations on the growing individual fundamental rogue components. This induced translation inhibits them forming an in-phase bound state of a higher-order rogue wave solution with a single maximum. Instead, they appear independently in their elementary form shifted in $z$ and $t$ as breakaway components.

\section{EVOLUTION DYNAMICS BEYOND ROGUE WAVE FISSION}

The disintegrated fundamental rogue waves develop on a background, and they remain highly susceptible to the effect of $\beta_{3}$ making their lifespan short lived. Consequently, this

(a)

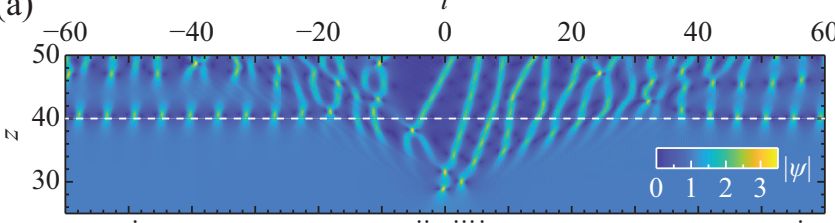

(b)

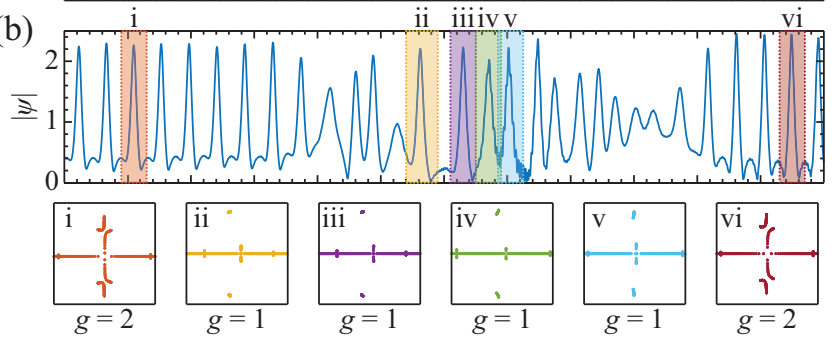

FIG. 8. (a) Amplitude evolution beyond the rogue wave fission. This is the same system we examined in Section III for the secondorder rogue wave when $\beta_{3}=0.18$. (b) Amplitude of the solution at $z=40$. The IST spectra of the six highlighted formations, labeled $\mathrm{i}-\mathrm{vi}$, are presented in the bottom panel. The real and imaginary parts of the eiegnvalues are plotted in the horizontal and vertical axes, respectively. 
transient rogue wave state quickly evolves into a bunch of solitons. Figure 8(a) is the extended evolution of the rogue wave fission. This is the same system we examined in Section III for the second-order rogue wave solution when $\beta_{3}=0.18$. Complex localized structures eventually follow after the fission. We identify the nature of these formations by applying the IST spectral analyses on six selected entities at $z=40$ as highlighted in Fig. 8(b). The IST spectra of the middle four, labeled ii-v, are of the genus $g=1$, representing soliton. On the other hand, the two that are away from the center, i.e., $i$ and vi, exhibit $g=2$, bearing the signature of either a rogue wave or a breather solution; the transverse periodic nature classifies it as a breather. Hence, the rogue wave fission triggers a group of solitons in the central region and breathers on the sides. In the neighborhood of a rogue wave fission, rogue waves, breathers, and solitons can coexist.

A common perception in rogue wave solution dynamics is that they appear from nowhere and disappear without a trace [31]. However, we demonstrate this may not always be the case. In a system that is prone to perturbations, the higher-order rogue wave solution may undergo fission first, ending up as rogue waves, breathers, and solitons rather than disappearing into the background.

\section{CONCLUSION}

In conclusion, we show that, similar to the higher-order soliton fission, an equivalent higher-order rogue wave fission also exists in a system weakly perturbed by the third-order dispersion. Using the second- and third-order rogue wave solutions, we reveal the breaking of the higher-order rogue wave, reducing it to their constituent parts. This new insight may prove to be useful for interpreting various nonlinear phenomena in optics, hydrodynamics, and other similar systems.

\section{ACKNOWLEDGMENT}

This work is supported by the Ministry of Education, Singapore (2019-T2-2-026).
[1] G. P. Agrawal, Nonlinear Fiber Optics, 5th ed. (Academic Press, Oxford, 2013).

[2] N. J. Zabusky and M. D. Kruskal, Interaction of "Solitons" in a Collisionless Plasma and the Recurrence of Initial States, Phys. Rev. Lett. 15, 240 (1965).

[3] A. R. Osborne, Nonlinear Ocean Waves and the Inverse Scattering Transform (Academic Press, London, 2010).

[4] F. Dalfovo, S. Giorgini, L. P. Pitaevskii, and S. Stringari, Theory of Bose-Einstein condenstation in trapped gases, Rev. Mod. Phys. 71, 463 (1999).

[5] M. J. Ablowitz and P. A. Clarkson, Solitons, Nonlinear Evolution Equations and Inverse Scattering (Cambridge University Press, Cambridge, 1991).

[6] D. H. Peregrine, Water waves, nonlinear Schrödinger equations and their solutions, J. Austral. Math. Soc. Ser. B 25, 16 (1983).

[7] V. E. Zakharov and A. B. Shabat, Exact theory of two-dimensional self-focusing and one-dimensional selfmodulation of waves in nonlinear media, J. Exp. Theor. Phys. 34, 62 (1972).

[8] A. V. Husakou and J. Herrmann, Supercontinuum Generation of Higher-Order Solitons by Fission in Photonic Crystal Fibers, Phys. Rev. Lett. 87, 203901 (2001).

[9] J. Herrmann, U. Griebner, N. Zhavoronkov, A. Husakou, D. Nickel, J. C. Knight, W. J. Wadsworth, P. St. J. Russell, and G. Korn, Experimental Evidence for Supercontinuum Generation by Fission of Higher-Order Solitons in Photonic Fibers, Phys. Rev. Lett. 88, 173901 (2002).

[10] A. Chabchoub, N. Hoffmann, M. Onorato, G. Genty, J. M. Dudley, and N. Akhmediev, Hydrodynamic Supercontinuum, Phys. Rev. Lett. 111, 054104 (2013).

[11] J. M. Dudley, F. Dias, M. Erkintalo, and G. Genty, Instabilities, breathers and rogue waves in optics, Nat. Photon. 8, 755 (2014).

[12] T. B. Benjamin, Instability of periodic wavetrains in nonlinear dispersive systems, Proc. R. Soc. London Ser. A 299, 59 (1967).
[13] T. Carmon, Nonlinear Optics and Solitons (Doctoral dissertation, Israel Institute of Technology, 2003).

[14] N. Akhmediev, V. Eleonsky, and N. Kulagin, Generation of periodic trains of picosecond pulses in an optical fiber: Exact solutions, J. Exp. Theor. Phys. 62, 894 (1985).

[15] N. Akhmediev, J. M. Soto-Crespo, and A. Ankiewicz, Extreme waves that appear from nowhere: on the nature of rogue waves, Phys. Lett. A 373, 2137 (2009).

[16] A. Chabchoub, N. P. Hoffmann, and N. Akhmediev, Rogue Wave Observation in a Water Wave Tank, Phys. Rev. Lett. 106, 204502 (2011).

[17] D. R. Solli, C. Ropers, P. Koonath, and B. Jalali, Optical rogue waves, Nature 450, 1054 (2007).

[18] P. Suret, R. E. Koussaifi, A. Tikan, C. Evain, S. Randoux, C. Szwaj, and S. Bielawski, Single-shot observation of optical rogue waves in integrable turbulence using time microscopy, Nat. Commun. 7, 13136 (2016).

[19] A. Ankiewicz, D. J. Kedziora, and N. Akhmediev, Rogue wave triplets, Phys. Lett. A 375, 2782 (2011).

[20] A. Ankiewicz, J. M. Soto-Crespo, M. A. Chowdhury, and N. Akhmediev, Rogue waves in optical fibers in presence of thirdorder dispersion, self-steepening, and self-frequency shift, J. Opt. Soc. Am. B 30, 87 (2013).

[21] B. Kibler, K. Hammani, C. Michel, C. Finot, and A. Picozzi, Rogue and shock waves in nonlinear dispersive media, Phys. Lett. A 375, 3149 (2011).

[22] M. Taki, A. Mussot, A. Kudlinski, E. Lourvergneaux, M. Kolobov, and M. Douay, Third-order dispersion for generating optical rogue solitons, Phys. Lett. A 374, 691 (2010).

[23] K. Trulsen and K. B. Dysthe, A modified nonlinear Schrödinger equation for broader bandwidth gravity waves on deep water, Wave Motion 24, 281 (1996).

[24] V. B. Matveev and M. A. Salle, Darboux Transformations and Solitons (Springer-Verlag, Heidelberg, 1991). 
[25] D. J. Kedziora, A. Ankiewicz, and N. Akhmediev, Classifying the hierarchy of nonlinear-Schrödinger-equation rogue-wave solutions, Phys. Rev. E 88, 013207 (2013).

[26] N. Akhmediev, Waves that appear from nowhere: Complex rogue wave structures and their elementary particles, Front. Phys. 8, 631 (2021).

[27] S. Randoux, P. Suret, and G. El, Inverse scattering transform analysis of rogue waves using local periodization procedure, Sci. Rep. 6, 29238 (2016).

[28] A. Chowdury, T. Gavara, and W. Chang, Emergence of breathers in non-linear pulse compression, J. Opt. 22, 085502 (2020).
[29] A. Tikan, C. Billet, G. El, A. Tovbis, M. Bertola, T. Sylvestre, F. Gustave, S. Randoux, G. Genty, P. Suret, and J. M. Dudley, Universality of the Peregrine Soliton in the Focusing Dynamics of the Cubic Nonlinear Schrödinger Equation, Phys. Rev. Lett. 119, 033901 (2017).

[30] F. Baronio, S. Chen, and S. Trillo, Resonant radiation from Peregrine solitons, Opt. Lett. 45, 427 (2020).

[31] N. Akhmediev, A. Ankiewicz, and M. Taki, Waves that appear from nowhere and disappear without a trace, Phys. Lett. A 373, 675 (2009). 